\title{
Collaborative Development of Teaching Scripts: An Efficient Faculty Development Approach for a Busy Clinical Teaching Unit
}

\author{
Valerie J. Lang, MD*, Alec B. O’Connor, MD, MPH${ }^{1}$, Amy Blatt, MD, Catherine Gracey, $\mathrm{MD}^{2}$
}

${ }^{1}$ Hospital Medicine Division, University of Rochester School of Medicine and Dentistry, Rochester, New York; ${ }^{2}$ General Medicine Division, University of Rochester School of Medicine and Dentistry, Rochester, New York.

BACKGROUND: Exemplary teachers have been shown to use teaching scripts, ways of organizing the content and instructional approach for commonly encountered teachable moments. This study describes a busy hospitalist unit's early experience with the collaborative development of teaching scripts.

METHODS: In 2010, during monthly workshops, 10 faculty members each prepared and presented a teaching script for a different commonly encountered diagnosis. Openended surveys assessing the impact on faculty were analyzed using an iterative approach. Changes in faculty self-efficacy, and the frequency and applicability of teaching were measured.

RESULTS: The program required 10 hours of attendance time and a mean of 4.3 hours for each faculty member who prepared a teaching script. No significant differences in quantity or applicability of teaching were detected, but faculty self-efficacy improved significantly. In addition, faculty described beneficial effects in their individual professional development, development of a shared mental model of professional responsibility, and interpersonal relationships. A majority of comments were positive; negative comments focused on the time required to prepare scripts, and apprehension about presenting to peers.

CONCLUSIONS: The program was an efficient approach to improve self-rated teaching skills, enhance professional development, and build collegiality among clinicianteachers. Journal of Hospital Medicine 2012;000:000-000 (C) 2012 Society of Hospital Medicine
Patient complexity, ${ }^{1}$ productivity, and documentation pressures have increased substantially over the past 2 decades. Within this environment, time for teaching is often limited. The same pressures which limit faculty members' teaching time also limit their availability to learn how to teach; faculty development efforts need to be both effective and efficient.

In a seminal study of exemplary clinical teachers, Irby discovered that expert teachers often developed and utilized "teaching scripts" for commonly encountered teachable moments. ${ }^{2}$ Teaching scripts consist of a trigger, key teaching points, and teaching strategies. ${ }^{2}$ A trigger may be a specific clinical situation or a learner knowledge gap identified by the teacher. The trigger prompts the teacher to select key teaching points about the topic (the content), and utilize strategies for making these teaching points comprehensible (the process). ${ }^{2}$ Through a reflective process, these expert teachers evaluated the effectiveness of each teaching session and honed their scripts over time. ${ }^{2}$

*Address for correspondence and reprint requests: Valerie J. Lang, MD, Hospital Medicine Division, University of Rochester School of Medicine and Dentistry, 601 Elmwood Ave, Box MED-HMD, Rochester, NY 14642; Telephone: 585-275-4912; Fax: 585-276-2212;

E-mail: Valerie_Lang@urmc.rochester.edu

Additional Supporting Information may be found in the online version of this article.

Received: March 20, 2012; Revised: July 8, 2012; Accepted: July 14, 2012

2012 Society of Hospital Medicine DOI 10.1002/jhm.1971

Published online in Wiley Online Library (Wileyonlinelibrary.com).
While additional reports have described the use of teaching scripts, ${ }^{3-5}$ we found no studies evaluating the impact of collaboratively developing teaching scripts. In the present study, we sought to understand faculty members' early experiences with a program of collaboratively developing teaching scripts and the impact on their self-efficacy with teaching about commonly encountered clinical conditions on attending rounds.

\section{METHODS}

Participants were the 22 internal medicine, or combined internal medicine and pediatrics (med-peds), hospitalists in a 750-bed university teaching hospital in upstate New York. Nine hospitalists worked for only 1 year (eg, chief residents and recent graduates awaiting fellowship training), and were present for half of the program year. All hospitalists conducted daily bedside attending rounds, lasting 1.5-2 hours, with a dual purpose of teaching the residents and students, and making management decisions for their shared patients.

Hospitalists were surveyed to identify 10 commonly encountered diagnoses about which they wanted to learn how to teach. The faculty development director (V.J.L.) conducted a 1-hour workshop to introduce the concept of teaching scripts, and role-play a teaching script. Nine hospitalists volunteered to write scripts for the remaining target diagnoses. They were provided with a template; example teaching script (see Supporting Information, Supplemental Content 1, in the online version of this article); and guidelines on writing scripts which highlighted effective clinical 
Teaching scripts are the organization of knowledge about a topic or learning situation into preplanned teaching points and strategies for how to teach. ${ }^{2}$ When writing your script, please consider the following (examples are given in italics):

\section{Identify the "Trigger", based on patient situation and learners' knowledge gaps}

a. A patient's symptom; need to develop a differential diagnosis Ex. Patient exhibiting confusion

b. Abnormal test result Ex. Anemia

c. Physical finding or bedside observation Ex. Patient has a foley catheter in place

d. Time to change management Ex. Switch from IV to oral antibiotics

\section{Target high-yield teaching points}

a. Real questions that residents and students face on a daily basis

Q: What's the most common hospital-acquired infection?

A: UTI (Note: Although central line infections, ventilator-associated pneumonia, and wound infections are important preventable infections, UTI's are more common. In fact, they are being targeted by Medicare as one of the hospital-acquired complications that they'll no longer pay for.)

b. Health systems, patient safety, and quality of care issues

$Q$ : Let's say you're the night float cross-covering another team's patients, and a nurse calls you asking to place a foley a patient who is getting lasix twice a day. She tells you that the patient is incontinent and has to use a walker to get to get to the bathroom. You know that "nursing convenience" is not a reason for placing a catheter. What are some alternatives to urinary catheters?

A: Scheduled toileting, bedside commodes, diapers, giving diuretics in the morning and mid-day instead of BID dosing, daily weights for monitoring of fluid status (often more accurate than "I's and $O$ 's").

c. Appropriate implementation of evidence-based guidelines

Q: Which antibiotic(s) would you choose to treat this pneumonia?

A: Recommend empiric antibiotics for community-acquired pneumonia.

d. Evidence-based physical diagnosis techniques

$Q$ : What is the sensitivity and specificity of various signs of congestive heart failure?

A: Elevated jugular venous pressure (positive LR 3.9; negative LR nonsignificant)

Positive abdomino-jugular test (positive LR 8; negative LR 0.3)

e. How you access the evidence/guidelines in real time

Encourage the team to access national guidelines at the point of care. Search "IDSA pneumonia guidelines" and choose the appropriate antibiotic regimen.

FIG. 1. Tips for developing teaching scripts with examples drawn from a variety of teaching scripts developed by hospitalists.

teaching principles for hospitalists, including: managing time with short scripts and high-yield teaching points, knowledge acquisition with evidence-based resources, self-reflection/insight, patient-centered teaching (identifying triggers among commonly encountered situations), and learner-centered teaching (identifying common misconceptions and strategies for engaging all levels of learners) (Figure 1). ${ }^{2,6}$ Faculty were encouraged to practice their scripts on attending rounds, using lessons learned to refine and write the script for presentation. Each script was presented verbally and on paper at a monthly 1-hour interactive workshop where lunch was provided. Authors received feedback and incorporated suggestions for teaching strategies from the other hospitalists. Revised scripts were distributed electronically.

Baseline surveys measured prior teaching and faculty development experience, and self-efficacy with teaching about the 10 target diagnoses, ranging from "Not confident at all" to "Very confident" on a 4-point Likert scale. Using open-ended surveys, we asked all of the hospitalists about their experiences with presenting scripts and participating in peer feedback, and the impact of the program on their teaching skills and patient care.

Because the learning objectives for each teaching script were determined by each script's author and were not known prior to the program, we were unable to assess changes in residents' and students' 
3. Identify evidence-based sources to support your teaching points

a. JAMA Rational Clinical Examination Series

b. McGee S. Evidence-Based Clinical Diagnosis

c. On-line resources

4. Keep script brief

a. Only 5-15 minutes long; longer if physical exam is included

b. These are not comprehensive reviews of a topic

\section{Describe your strategy for teaching}

a. Keep it interactive

Q. Aside from UTI's, what are other complications of urinary catheters?

(Note: Start by asking the students, then the interns, and then the senior resident.)

A. Urethral tears Retention from urethral swelling

Incontinence from bladder shrinkage after the catheter is removed Delirium-especially in demented patients

Falls (catheters are "one-point restraints")

b. Use analogies, visual representations, mnemonics, point-of-care resources, other activities

Let's run the list again. Are there any patients on the team whose catheters we can remove today? (Note: Let the students tell you about their patients, then the interns. Stand while they enter the orders to discontinue the foley-it will feel like a great accomplishment.)

c. Explain how you involve learners at different levels

$Q$ : Without going into the rooms and looking, how many patients on the team have urinary catheters right now? (Note: Start by asking the students, then the interns, then the senior resident individually about the patients they're following.)

A: Students are most likely to know whether or not a catheter's in place; the intern's next most likely, then senior resident. Attendings are usually in the dark.

d. Identify common misconceptions encountered among students and residents "Incontinence" and "inability to ambulate" are often mentioned, but these are not indications for urinary catheters. Just think of all the babies in the nursery who are incontinent and non-ambulatory. Also, many of our older patients are chronically incontinent and manage without catheters outside the hospital. "Nursing convenience" is not an indication either.

\section{Practice your script with your team(s)}

\section{Demonstrate your script to other hospitalists for feedback}

\section{Revise your script and submit for the on-line Hospital Medicine Division Teaching Manual}

FIG. 1. Continued.

knowledge directly. As a surrogate measure, we surveyed students, residents, and faculty regarding how often the hospitalist taught about the 10 target diagnoses and whether teaching points were applicable to current or future patients. We administered the surveys online weekly for 8 weeks before and after the program. Residents and students were notified that participation had no impact on their evaluations. They received a $\$ 2.50$ coffee gift card for each survey. The study received an exemption from the university's Institutional Review Board.
The number of teaching episodes per week related to the target diagnoses was averaged across survey weeks. Student $t$ tests were used to compare results before versus after the intervention, and $95 \%$ confidence interval (CI) calculated. We considered $P<$ 0.05 to be statistically significant. Data were analyzed using SAS version 9.2 (Cary, NC).

Qualitative data were analyzed by coding each statement, then developing themes using an iterative process. Three investigators independently developed themes, and met twice to review the categorization of each 
statement until consensus was achieved. Two of the investigators were involved in the program (V.J.L. and A.B.) and one did not participate in the workshops (C.G.).

\section{RESULTS}

The 22 faculty had an average of 5 years' experience as hospitalists (range 0.8-24 years). Previous experience formally learning how to teach ranged from 0 to 150 hours (average 33.1 hours; median 15 hours). A mean of 9.4 hospitalists attended each of the $10 \times 1$-hour workshops. Script writers estimated that scripts required a mean of 4.3 hours to prepare. A total of 105 (59\%) resident/student and $22(55 \%)$ faculty surveys were returned preintervention, and $83(47 \%)$ resident/student and 19 $(48 \%)$ faculty surveys were returned postintervention. There were no significant differences in the number or applicability of teaching events from before to after the program. Faculty self-efficacy with teaching was available for 7 of the 10 diagnoses, and increased from a mean of $3.26(\mathrm{n}=77)$ preintervention to $3.72(\mathrm{n}=52)$ postintervention $195 \% \mathrm{CI}$ for the difference in means $0.35-0.51 ; P<0.0001)$.

A total of $8(80 \%)$ script-writers and $5(42 \%)$ non-writers responded to the qualitative survey, and 77 comments were coded. Three major themes and 8 subthemes were identified (for representative comments, see Supporting Information, Supplemental Content 2, in the online version of this article). The major theme of individual professional development related especially to the personal satisfaction of researching a topic and becoming a "local expert." While most comments were positive, 2 described apprehension about presenting to peers. Fifteen comments specifically addressed the development of teaching skills, 13 positive and 2 neutral. Some focused on strategies consistent with the teaching script framework, including recognizing teachable moments and the importance of preparation for teaching. Others focused on changes in teaching style, shifting to a more interactive method and involving multiple levels of learners. Others revealed that participants adjusted the content of their teaching, adding new material and changing the focus to important clinical pearls. Another subtheme was the impact on clinical care and medical knowledge base. Of the 11 comments, 7 were positive and emphasized the development of a framework for making decisions, based on an understanding of the evidence behind those decisions. Four were neutral, noting that care of patients had not changed. Two comments remarked on the time invested in developing teaching scripts. A second major theme was the development of a shared mental model of professional responsibility. This was demonstrated by comments relating to participants' motivation for learning, and development or strengthening of responsibility for teaching. The third major theme described interper- sonal relationships among colleagues. Four commented on how the opportunity to see how others teach led them to appreciate the diversity of approaches, while 14 focused on collegiality among the faculty. Thirteen of these identified an increased sense of community and camaraderie, while one was neutral.

\section{CONCLUSIONS}

We had successful early experience with a faculty development intervention that involved hospitalists in creating and implementing teaching scripts related to commonly encountered diagnoses. The intervention was time- and resource-efficient. Following the intervention, we found increased faculty self-efficacy and beneficial effects in several domains related to professional development and satisfaction. We found no significant difference in the frequency or applicability of teaching about the targeted diagnoses.

In addition to the formal program evaluation results, we learned several additional lessons informally. Faculty who developed scripts had varying levels of familiarity with evidence-based approaches to teaching. Some faculty requested to have their scripts reviewed by the program leader before presentation, and small revisions were made, emphasizing use of the tips included in Figure 1. Using volunteers, rather than assigning the responsibility for script development, ensured that we had a group of enthusiastic participants. In fact, several hospitalists volunteered to write additional scripts the following year.

This program used a conceptual framework of best practices, namely evidence-based principles of effective faculty development for teaching in medical education. $^{7}$ Different instructional methods were utilized: experiential learning was simulated by demonstrating scripts; the reasoning underlying scripts was provided; feedback was provided; and scripts were provided in written, electronic, and verbal formats. Allowing hospitalists to choose which script to develop gave them a chance to showcase an area of strength or explore an area of weakness, a feature of self-directed learning. Focusing scripts on common diagnoses and easily identifiable triggers enhanced the functional value of the workshops. By having each hospitalist develop a script with input from each other, the unit built a body of knowledge and skill, enhancing collegiality and building a community of learners. Studies of other longitudinal faculty development programs have found that they create a supportive, learner-centered environment that fosters a sense of commonality and interdisciplinary collegiality. ${ }^{8,9}$

Other faculty development initiatives specific to hospitalists have been described, several focusing on the care of geriatric patients, ${ }^{10-12}$ and one focusing on general academic development. ${ }^{13}$ While effective, these programs depended on a few individuals to develop the materials, and one required extensive time 
away from clinical duties for attendance. ${ }^{12}$ By sharing responsibility for developing teaching scripts, our program was efficient to conduct and capitalized on unique contributions from each faculty member.

This study has several limitations. While we attempted to quantify the amount and applicability of teaching, we were not able to account for the number of inpatients on the teams who had the diagnoses for which teaching scripts had been developed. It was impossible to determine whether these diagnoses were the most important topics to discuss on rounds. Because learning objectives were developed as each script was written, we were unable to assess changes in resident and student knowledge or patient outcomes. The study was conducted at a single center with interested faculty.

Future studies are needed to compare the effectiveness of collaborative teaching script development programs with other faculty development initiatives, and assess the impact on downstream outcomes, such as learners' decision-making, patient outcomes, and faculty retention.

\section{Acknowledgements}

The authors thank the members of the University of Rochester Hospital Medicine Division.

Disclosures: Funding: University of Rochester School of Medicine and Dentistry, Office of the Dean of Faculty Development-Medical Education. Conflicts of interest: Nothing to report. Ethics approval: Exemption given by the University of Rochester Research Subjects Review Board. Previous presentations: University of Rochester Faculty Development Colloquium, June 2011.

\section{References}

1. DeFrances CJ, Lucas DA, Bule VC, Golosinskly A. 2006 National hospital discharge survey. Centers for Disease Control and Prevention. Natl Health Stat. 2008;5:1-20.

2. Irby DM. How attending physicians make instructional decisions when conducting teaching rounds. Acad Med. 1992;67(10):630-638.

3. Marcdante KW, Simpson D. How pediatric educators know what to teach: the use of teaching scripts. Pediatrics. 1999;104:148-150.

4. Richardson WS, Wilson MC, Keitz SA, Wyer PC. Tips for teachers of evidence-based medicine: making sense of diagnostic tests using likelihood ratios. J Gen Intern Med. 2006;23(1):87-92.

5. Wiese J. Teaching scripts for inpatient medicine. In: Wiese J, ed. Teaching in the Hospital. ACP Teaching Medicine Series. Philadelphia, PA: American College of Physicians (ACP); 2010.

6. Fromme HB, Bhansali P, Singhal G, Yudkowsky R, Humphrey H, Harris I. The qualities and skills of exemplary pediatric hospitalist educators: a qualitative study. Acad Med. 2010;85(12):1905-1913.

7. Steinert Y, Mann K, Centeno A, et al. A systematic review of faculty development initiatives designed to improve teaching effectiveness in medical education: BEME guide no. 8. Med Teach. 2006;28(6): 497-526.

8. Pololi LH, Frankel RM. Humanising medical education through faculty development: linking self-awareness and teaching skills. Med Educ. 2005;39:154-162.

9. Gruppen LD, Simpson D, Searle NS, Robins L, Irby DM, Mullan PB. Educational fellowship programs: common themes and overarching issues. Acad Med. 2006;81:990-994.

10. Mazotti L, Moylan A, Murphy E, Harper GM, Johnston CB, Hauer KE. Advancing geriatrics education: an efficient faculty development program for academic hospitalists increases geriatrics teaching. $J$ Hosp Med. 2010;5(9):541-546.

11. Lang VJ, Clark NS, Medina-Walpole A, McCann R. Hazards of hospitalization: hospitalists and geriatricians educating medical students about delirium and falls in geriatric inpatients. Gerontol Geriatr Educ. 2008;28(4):94-104.

12. Podrazik PM, Levin S, Smith S, et al. The curriculum for the hospitalized aging medical patient program: a collaborative faculty development program for hospitalists, general internists, and geriatricians. J Hosp Med. 2008;3:384-393.

13. Sehgal NL, Sharpe BA, Auerbach AA, Wachter RM. Investing in the future: building an academic hospitalist faculty development program. J Hosp Med. 2011;6(3):161-166. 